\title{
A combined framework for the life cycle assessment and costing of food waste prevention and valorization: an application to school canteens
}

Fabio De Menna ${ }^{1}$, Jennifer Davis ${ }^{2}$, Karin Östergren $^{2}$, Nicole Unger ${ }^{3}$, Marion Loubiere ${ }^{4}$ and Matteo Vittuari ${ }^{{ }^{*}}$ (D)

* Correspondence: matteo.vittuari@ unibo.it

${ }^{1}$ Department of Agricultural and Food Sciences, University of Bologna, Viale Giuseppe Fanin 50, 40127 Bologna, Italy

Full list of author information is available at the end of the article

\begin{abstract}
About one third of global edible food is lost or wasted along the supply chain, causing the wastage of embedded natural and economic resources. Life cycle methodologies can be applied to identify sustainable and viable prevention and valorization routes needed to prevent such inefficiencies. However, no systemic approach has been developed so far to guide practitioners and stakeholders. Specifically, the goal and scoping phase (e.g. problem assessed or system function) can be characterized by a large flexibility, and the comparability between food waste scenarios could be not ensured. Within the Horizon2020 project Resource Efficient Food and dRink for the Entire Supply cHain, this study aimed to provide practitioners with guidance on how to combine life cycle assessment and environmental life cycle costing in the context of food waste. Recent literature was reviewed to identify relevant methodological aspects, possible commonly adopted approaches, main differences among studies and standards and protocols, main challenges, and knowledge gaps. Basing on this review, an analytical framework with a set of recommendations was developed encompassing different assessment situations. The framework intends to provide a step by step guidance for food waste practitioners, and it is composed of a preliminary section on study purpose definition, three decision trees - respectively on assessment situation(s), costing approach, and type of study (footprint vs. intervention) - and two sets of recommendations. Recommendations can be applied to all levels of the food waste hierarchy, stating a generic order of preference for handling food chain side flows. This consistent and integrated life cycle approach should ensure a better understanding of the impact of specific interventions, thus supporting informed private and public decision making and promoting the design of sustainable and cost-efficient interventions and a more efficient food supply chains.
\end{abstract}

Keywords: Life cycle costing, Life cycle assessment, Food waste, Food loss, E-LCC, LCA, REFRESH

\section{Introduction}

Global estimates suggest that about one third of edible food produced is lost or wasted along the supply chain due to several factors, from technological to behavioural ones (Gustavsson et al. 2011). This vast inefficiency has a relevant hidden burden, constituted by the direct and indirect wastage of embedded natural and economic resources

(c) The Author(s). 2020 Open Access This article is distributed under the terms of the Creative Commons Attribution 4.0 International License (http://creativecommons.org/licenses/by/4.0/), which permits unrestricted use, distribution, and reproduction in any medium, provided you give appropriate credit to the original author(s) and the source, provide a link to the Creative Commons license, and indicate if changes were made. 
consumed within food systems. FAO recently estimated the full cost of food waste at US\$2.6 trillion, when also environmental and social impacts are monetized (FAO 2013, 2014).

The magnitude of and the urgency to address food waste pushed the United Nations to include among the Sustainable Development Goals (SDG) a specific target (SDG12.3) to halve the per capita global food waste at the retail and consumer level and to reduce losses along production and supply chains. The European Commission championed this cause, aligning itself to the UN target in the Circular Economy package, which identifies food waste as one of five focus waste streams and sets measures aimed at the reduction and reuse of food waste, from production to consumption and end of life (European Commission 2015).

Food waste prevention is often identified as a crucial strategy to save embedded resources, potentially feed more people, and reduce the environmental pressure of food systems (Kummu et al. 2012; FAO 2013; Vittuari et al. 2016). Similarly, food waste valorization could allow the recovery of several resources, from animal feed and energy, to fertilizers and high-value compounds. However, the transformations required for preventing and valorizing food waste will have their own economic and environmental impacts. Private and public decision-makers will require robust, consistent, and science-based approaches to inform their interventions with reliable figures.

Life cycle approaches-such as life cycle assessment (LCA) (ISO 2006; EC JRC 2010) and life cycle costing (LCC) (Hunkeler et al. 2008; Swarr et al. 2011)-are often proposed as useful tools for the analysis of economic and environmental impacts of waste prevention, valorization, and management. By allowing comparative analysis within and across the waste hierarchy, the combined use of LCA and LCC could promote a better understanding of the impacts of specific interventions. Specifically, it would allow identifying possible trade-offs between solutions and sustainability pillars, win-win strategies, effective policy measures, and incentives (De Menna et al. 2018). In addition, this would be in agreement with the provisions set by the European Commission directive on waste (European Parliament and Council 2008).

Life cycle assessment (LCA) is well established in studying environmental impacts of food waste (FW), but no systemic approach for practitioners has been developed to date (Notarnicola et al. 2016; Unger et al. 2016; Gruber et al. 2016; Corrado et al. 2017). Life cycle costing (LCC) has been applied to FW only in a limited number of studies and without consistent practices (De Menna et al. 2018). In general, the goal and scoping phase of these studies presents a large variability in terms of problem(s) assessed or system function(s) identified. The outcome is often a spectrum of incoherent methodological choices. Therefore, results from FW-related studies are not always comparable and could easily lead to misinterpretation by non-experts. Even experienced LCA and LCC practitioners could experience challenges in making specific methodological choices related to FW or to the development of an integrated approach for LCA and LCC. Both the International Reference Life Cycle Data System (ILCD) handbook (EC JRC 2010) and the guidance on product environmental footprints (PEF) (EC 2013) focus on the driving product of a system, rather than its waste flows.

The EU Horizon2020 funded the project Resource Efficient Food and dRink for the Entire Supply cHain (REFRESH) aimed to contribute to food waste reduction throughout the food supply chain and evaluate the environmental impacts and life cycle costs. 
Within the REFRESH project, the objective of this study was to develop a consistent approach for combining LCA and LCC, specifically to assess impacts of prevention of resource inefficiencies, new/novel valorization options, and waste handling options relating to side flows in the food supply chain. The following paragraphs will describe the methods that were followed to develop the framework, present an overview of the approach and related decisional trees, and demonstrate its potential application with an example on school canteens.

\section{Methodology}

In the first step of the REFRESH project, literature on LCA and LCC of food waste was systematically reviewed to identify relevant methodological aspects. Sources included existing LCA and LCC standards documentation, academic journal papers, policy guidelines, and case studies on FW. The specific aim of the review was to identify and classify existing approaches and highlight the main differences among studies, standards, and protocols, as well as outstanding challenges and knowledge gaps. Detailed results and list of sources of this review were presented in two REFRESH reports (De Menna et al. 2016; Unger et al. 2016).

As far as LCA of food systems is concerned (Unger et al. 2016), several sources provide guidance on environmental assessment of food systems, but they leave a lot of room for LCA scoping. For example, standards and generic guidelines cover many products and services, but they do not provide step-by-step instructions on how to perform an LCA. Thus, food waste stakeholders, who may have a deep understanding of their system, but only a generic knowledge of LCA, would not find straightforward answers to specific modelling questions. Common issues included:

- Selection of attributional or consequential types of study;

- Establishing appropriate and coherent functional unit (FU) and system boundaries connected to the question(s) being addressed;

- Addressing multi-functionality (allocation vs. system expansion) and product substitution issues;

- Selection of impact indicators and interpretation of results.

As far as LCC is concerned, the review identified a widespread array of existing definitions and approaches (De Menna et al. 2016). Additionally, only a limited number of case studies were retrieved. These are mostly concerned with municipal FW management, and only a few included prevention issues. The following challenges could be identified:

- Lack of detailed recommendations for combining LCC and LCA;

- Use of LCC in case of consequential LCA studies;

- Differences between cut-off levels/scoping boundaries;

- Relevant cost categories for FW;

- Inclusion of economic impact indicators other than cost.

Based on the identified challenges, a specific framework was developed with the aim of providing a step-by-step assessment guidance for food waste practitioners (Davis 
et al. 2017). The framework is composed of an introductory section on study purpose definition, three decision trees-respectively on assessment situation(s), costing approach, and type of study (footprint vs. intervention) - and two sets of recommendations.

The framework was reviewed by selected LCA, LCC, and FW experts and practitioners within the REFRESH consortium and tested with selected case studies within the project.

\section{Results and discussion}

The overall structure of the framework is provided in Fig. 1.

In the first section of the framework, the assessor needs to identify the purpose of the study. The International Organization for Standardization (ISO) standard provides information for several methodological items describing the goal and scope of an LCA study (ISO 2006). However, as highlighted by Unger et al. (2016), when carrying out a FWrelated study, there is a set of aspects to be clarified relating to modelling framework for the study (consequential or attributional), functional unit and system boundaries, handling multi-functional processes, when and how to attribute burden to the side flow, how to identify a replaced product, and what environmental impact indicators to focus on.

Therefore, in the current framework, the main elements to include in the description of the purpose of the study include:

- Indication of the product/process under analysis (what product, what waste flow, and what characteristics);

- Decisions on:

Whether the goal is to assess a current situation or changes towards an alternative scenario,

Whether prevention is included or foreseen, and

Whether some value is or will be involved in the management of the side flow.

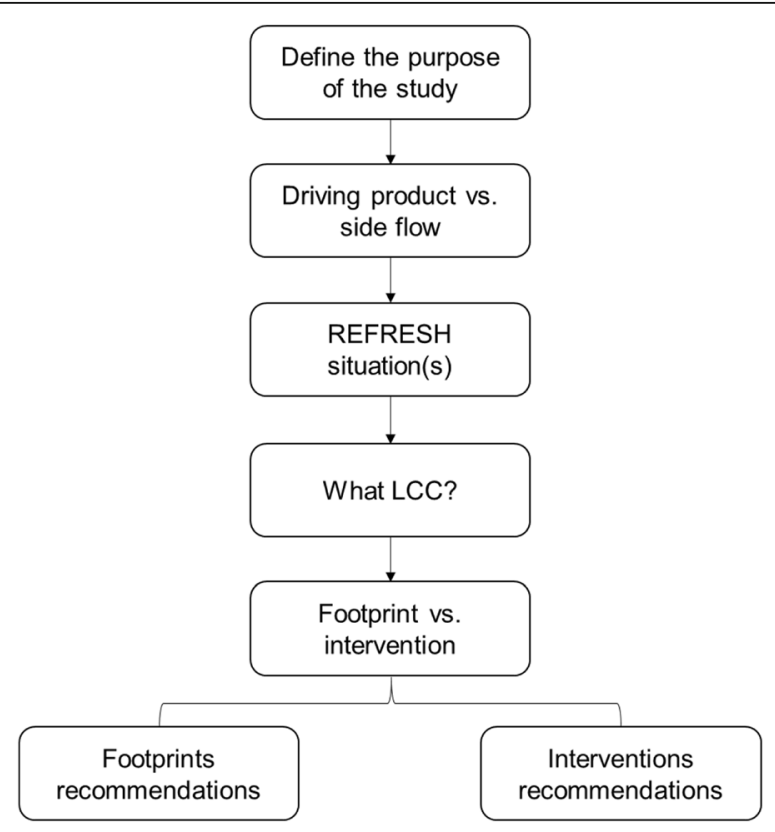

Fig. 1 Framework structure 
Once the purpose of the study is established, the next step is to identify whether the flow under study should be considered a driving product or a side flow (Davis et al. 2017). A flow of the food supply chain can be characterized as a driving product whenever it represents the main reason for the supply chain to exist. This means that in some agro-food processes, there can be several driving products, all of which are justifying a certain share of the studied system. On the contrary, any wasted edible and inedible part of food-including wasted flows of driving product(s) — can be defined as side flow. The main difference with the driving product is that an assessor would like to minimize it, rather than producing more of it. In the REFRESH framework, since the focus is on impacts of food waste, guidance and recommendations are provided with reference to the assessments of side flows. Instead, no guidance is given on the evaluation of driving products.

Once the purpose and subject of the study are defined, it is important to identify the potential situation(s) to assess. A practitioner could for example want to evaluate impacts of a specific prevention measure or estimate potential costs and impacts of a prospective pilot plant. While side flows and life cycle stages may be different, any given assessment situation will share certain methodological commonalities.

To categorize systems suitable for comparative assessment, the concept of "REFRESH situations" (RS) has been developed (De Menna et al. 2016; Unger et al. 2016; Davis et al. 2017). The RS include the following: prevention of side flow (RS 1), side flow valorization (RS 2), valorization as part of waste management (RS 3), and end-of-life treatment (RS 4). REFRESH situations can take place at any point/process within the life cycle and within the remit of any stakeholder (including consumers) and are independent of the perspective taken, i.e. of the producer of side stream or the receiver. For each REFRESH situation, specific recommendations on the setting of system boundary, functional unit(s), and handling of multi-functionality in relation to the stated problem are provided (besides other aspects). This categorization was then translated into a decision tree meant to help practitioners in the selection of relevant situations.

Another decision tree was developed for the selection of the most appropriate costing approach. Life cycle costing is a rather consolidated methodology to assess the total costs related to the life span or cycle of a product or service. As emphasized in De Menna et al. (2016, 2018), LCC was only recently applied to food waste management and valorization studies, to integrate LCA with an accounting or economic tool. In this regard, Hunkeler et al. (2008) suggested three different approaches: conventional, environmental, and societal LCC. The major differences are related to the stakeholders included in the assessment, the system boundaries, and the potential integration with LCA. The framework adopts this distinction and provided guidance to users to identify the appropriate method. Specifically, if only internal costs are assessed, a conventional LCC (C-LCC) is suggested. This approach is traditionally used to analyse long-term projects beyond the initial investment costs, by including also operating and maintenance costs emerging during the life span. Disposal costs are included only as long as they are sustained by the assessor. C-LCC is usually not carried out in integration with LCA, but it can be used when system boundaries are cradle to gate and only one actor is covering for all costs.

If the environmental and costing dimensions are part of the same assessment, it is possible to implement different approaches according to the stakeholders included and 
the type of integration with LCA. The assessor should decide whether to include costs for all the stakeholders that may be affected by the analysed system also through externalities, such as society and governments. Such perspective is coherent with a societal LCC (S-LCC) approach. In S-LCC, costs affecting every stakeholder, both directly and indirectly through externalities (e.g. environmental impacts), are assessed. As argued by Martinez-Sanchez et al. (2016), an S-LCC covers all externalities that can be monetized. Therefore, the integration with a complementary LCA support the S-LCC in identifying and quantifying relevant externalities to be then monetized, although a standard approach for monetization is still under development (ISO 14008). If the assessor decides to limit the analysis to relevant stakeholders of the analysed system that are directly sustaining costs (e.g. different actors of the supply chain), then the recommended approach is the environmental LCC (E-LCC), accompanied by a complementary LCA evaluating the environmental dimension. As defined by Hunkeler et al. (2008), E-LCC is a LCC approach that "summarizes all costs associated with the life cycle of a product [including] those involved at the end of life; these costs must relate to real money flows. Externalities that are expected to be internalised [...] must also be included". Thus, ELCC should not be considered a weighting method, so as an approach translating environmental externalities into monetary value as in S-LCC, but a costing method that can be integrated with LCA and therefore that shares the same functional unit and system boundaries. E-LCC covers external costs already internalized (e.g. taxes) and those that are expected to be internalized in the future, for instance the introduction of a $\mathrm{CO}_{2}$ tax or a tax on fats that will internalize external costs. E-LCC can serve several purposes. Typologies of goal and scope of an E-LCC provided by Hunkeler et al. (2008) and reviewed in De Menna et al. (2016) include:

- Assessment of total costs for an actor: similarly, to a C-LCC, a business can use an E-LCC for the evaluation of costs related to different food waste scenarios identifying insights for both internal firm management and investment plans;

- Evaluation of competitiveness: to evaluate the potential marketability of a product (e.g. bioplastic from food waste), a firm can use an E-LCC approach to assess both its cost and benefits and those that will be incurred by consumers during the use/ ownership of a product;

- Identification of trade-offs or win-win solutions: coupled with an LCA, an E-LCC can support businesses to identify the least expensive environmental measure or the breakeven between costs and environmental impacts;

- Estimation of value-added and supply chain effects: differences in terms of valueadded or cost distribution along the supply chain can be estimated with an E-LCC approach.

This method is covered by the recommendations within the framework as far as side flow valorization and management are regarded.

Subsequently to RS and LCC approach choice, the last decision tree is related to the modelling approach to be used. The general modelling framework influences several other choices. There is a consistent debate on the distinction between attributional LCA (ALCA) and consequential LCA (CLCA). The main differences are summarized in Table 1. 
Table 1 Attributional and consequential LCA

\begin{tabular}{|c|c|c|}
\hline & ALCA & CLCA \\
\hline Goal & $\begin{array}{l}\text { Assessment of the impact of a functional unit, } \\
\text { with representative data in a defined geography } \\
\text { and socio-economic conditions }\end{array}$ & $\begin{array}{l}\text { Assessment of the effect of changes } \\
\text { on one system in a specified horizon } \\
\text { (interventions) }\end{array}$ \\
\hline Comparative & Possible & Always \\
\hline FU and scale & $\begin{array}{l}\text { FU (often one unit) has the same impact as } \\
\text { the previous or next, independent of scale }\end{array}$ & $\begin{array}{l}\text { FU represent the scale of the intervention } \\
\text { as it will determine processes affected }\end{array}$ \\
\hline
\end{tabular}

Considering the related differences, the framework builds on the distinction between attributional and consequential approaches and coherently identifies two types of studies: footprint studies and intervention studies. In the first case, studies that are evaluating the impact deriving from a product (e.g. providing a snapshot of a valorized product from a side flow) and are not focusing on the consequences on other parts of the economy can be defined as footprint studies of side flows. Therefore, the modelling approach in this case is the attributional one. In such case, the study is usually referring all impacts on the valorized product from the side flow. Footprint studies can be carried out only for RS2, 3, and 4.

On the contrary, if the aim of the assessor is to estimate the effects of certain changes in a system (e.g. changing from waste management to the prevention of a side flow), then an intervention study should be carried out. In this case, the end/future situation (including RS1) is compared to the current situation and impacts of all changes are evaluated. Therefore, the modelling approach is consequential and the functional unit is constituted by the prevented/valorized/managed side flow.

Finally, the framework provides two sets of recommendations on selected issues for LCA and LCC, respectively, for footprint and intervention studies. In specific, indications are provided for:

- Functional unit

- System boundaries

- Multifunctionality

- Cut-off principles

- LCA inventory and cost modelling

- Impact assessment (separate and combined)

- Interpretation of results

\section{Framework application}

The application of the framework is presented through a fictious example, inspired by the existing literature on school canteens (Cerutti et al. 2016; Boschini et al. 2018; Garcia-Herrero et al. 2019) and representing a realistic situation. It is assumed that a generic school canteen catering company aims at evaluating the environmental and economic consequences of an intervention aiming at the prevention of serving and plate waste during school lunch.

The current situation is including the serving of about 200,000 meals per year in the schools of a medium-sized municipality. Serving and plate waste constitutes almost $25 \%$ of the food mass, equivalent to $30 \mathrm{t}$ of organic waste per year. This food waste is 
disposed of through a large municipal biogas facility, generating biogas for electricity and digestate. The school is charged a fee for waste collection.

In the perspective scenario, the catering company, in agreement with the municipal council, would like to implement in the school a prevention measure by reducing the amount of served food. This measure is predicted to have an effect of $30 \%$ reduction of food waste. The municipality is also planning to introduce a "food waste carbon tax" charging an extra fee for the disposal of wasted food, calculated on the embedded $\mathrm{CO}_{2}$ eq emissions of food and based on the carbon emission trading system.

The specific aim of the analysis for the assessor (the catering company) is the assessment of the costs for all the engaged parties and the identification of the environmental impact related to this change.

\section{Step 1: Phrase the question of the study and identify the audience for the result}

The focus of the study is on the change in economic costs and environmental impacts for the catering company, the school, and the biogas operator, which could derive from a reduction in the amount of organic waste sent to municipal waste handling, obtained by preventing serving and plate waste. The result of the study should support the catering company and the municipality in the decision to undertake such intervention.

\section{Step 2: Establish REFRESH situations}

The REFRESH situation decision tree allows to establish if the study explores a side flow (not a deriving product) thanks to the principle "the less, the better' applied for discarded products. The current scenario is represented by RS3 (valorization as part of waste management) since the catering company and the school just dispose of food waste and pays a fee for its collection, but the biogas producer extracts some value out of the treatment. The perspective scenario is represented by RS1 (prevention) since the reduction of the amount of food waste sent to the biogas facility would guarantee a saving in the resources needed (purchased) for the disposal of such waste.

\section{Step 3: Footprint or intervention study?}

Since the catering company will take its decision on the basis of the results of the study, an intervention modelling approach is recommended. Considering the scale of the flow (30,000 t per year), the intervention might influence the operations of the municipal biogas facility leading to some consequences in the background processes. Thus, the modelling system expansion should be considered when accounting for "additional" functions from the system, using marginal market data for processes in the background system (e.g. how electricity and digestate from that biogas plant will be substituted).

\section{Step 4: E-LCC appropriate?}

The catering company wants to assess both environmental and cost. In addition, only stakeholders directly involved in the supply chain will be considered (the food ingredient suppliers, the catering company, the school, and the biogas operator). No other stakeholders external to this chain are included, so it is recommended to select an ELCC approach. 
Step 5: FU, SB, cut-off, and handling multi-functionality

While in the goal and scope stage of an LCA study, several aspects must be included to comply with international standards; this example is focusing only on the aspects of FU, SB, cut-off, and multi-functionality. Since the aim of the study would be to assess the effects of moving from RS 3 to RS 1 (prevention), then the FU and SB should include the supply chain of the driving product (ingredient production, meal production, and consumption) and not only the food waste management chain. Specifically, the FU should be the 200,000 meals, over a time period of 1 year, in the time horizon 20182023. Details about the composition of the driving product and side flow should also be provided.

The inclusion of the supply chain of the driving product is also needed because food waste prevention actions might cause a reduction of produced food in the upstream processes. Such perspective would thus allow to analyse such consequences.

For the E-LCC, system boundaries should be coherent with LCA, e.g. considering the same life cycle stages. Catering costs must be fully inventoried. Market prices can be used for the production of inputs for processes (e.g. fuels and chemicals). Costs of cultivation and transport stages can be estimated using market prices of purchased food/ service as a proxy or with further detail. This latter case is desirable if internal costs for suppliers are changing (e.g. because different purchasing levels by catering company could lead to different production by suppliers). The same options are also valid for downward processes related to biogas. Avoided impacts from system expansion can be estimated either through revenues from electricity and digestate or market price of identified substituted products.

Cut-off can be different for LCA and LCC. In the first case, it should exclude processes such as production of seeds and seedlings, transport of personnel, transport of fertilizers, production of equipment, buildings, and vehicles, due to their limited influence on final results. In the E-LCC, considering that worker training and hours as well as indirect costs might change, the cut-off should include also items not related to LCA flows, adopting a financial relevance criterion.

As for the multifunctionality, current systems deliver also biogas and digestate, which are replacing respectively natural gas and mineral fertilizer. In the perspective scenario, prevention of serving and plate waste would hardly have the magnitude to influence the market but could have some local consequences (e.g. municipal biogas facility operating at a reduced rate). Average market data for substitute product and to model the impact should be used in both scenarios, including also avoided costs.

\section{Step 6: LCl data}

Data collection should focus on primary data for catering and service processes and the production of biogas. Food production and transport, replaced production of fuel and fertilizer, and transport and energy production impacts could be modelled using secondary data.

Cost modelling should categorize costs by type of costs, life cycle stages, and specific cost items. Primary cost data related to catering must be used while market prices can be used as proxy of other stages (see above). Considering the nature of catering operation (e.g. potential production of meals for other services), some indirect costs should 
be allocated to the FU, using a specific factor, such as the share of meals/turnover of FU over the total. Contrarily, no discounting is mandatory due to the relatively short time horizon of the intervention. Depreciation of plants and machineries can be used. Since the introduction of a "food waste carbon tax" is anticipated, the external cost of embedded CO2eq emissions in wasted food should be encompassed by the inventory and the impact assessment. Finally, cost modelling should focus on the relevant actors of the supply chain that can be affected by the intervention. In the current scenario, the caterer is purchasing food (including its transport) at market prices, thus bearing all those costs. Municipal waste management company is bearing all costs and benefits related to food waste disposal. In the future scenario, agricultural producers and the transport company will likely have to cope with a reduction of revenues, and the municipal waste management company could operate at a reduced rate, so it should be considered as a cost bearer of the system.

\section{Step 7: LCIA}

Since food production is included, impact indicators suggested are GWP, water depletion, depletion of fossil resources, terrestrial and aquatic eutrophication, acidification, and land transformation. In the E-LCC, it is recommended to assess costs and their changes for the different cost bearers (cost distribution), according to the categories and details included in the model. A portfolio of total results and contributions of life cycle stages/cost bearers could be useful to present results in a combined way. A plot graph could show percentage changes of moving from RS 3 to RS 1 for different indicators, e.g. GWP and total costs. If the catering company would prefer to have a single score, weighting and sum of LCA and E-LCC scores should be carried out to avoid double counting of externalities (e.g. "food waste carbon tax").

\section{Conclusions}

This study aimed to develop a consistent approach, combining LCA and LCC specifically to assess impacts of prevention of resource inefficiencies, new/novel valorization options, and waste handling options related to side flows of the food supply chain.

Several challenges and methodological issues were identified through a literature review of LCA and LCC of FW. A specific framework was then developed to provide a step-by-step assessment guidance for food waste practitioners. Recommendations are provided on the study purpose definition, specific typologies of assessment situation(s), costing approach and methods, and combined LCA and LCC modelling.

Recommendations are applicable to all levels of the waste hierarchy stating a generic order of preference for handling of side flows. The food waste hierarchy provides guidance on the identification and selection of the most preferred interventions.

This approach can support informed decision-making and in the long term promote the design of sustainable and cost-efficient interventions and more resource-efficient food supply chains. Finally, food loss and waste reduction present also relevant social (e.g. availability of food) and political implications that should be considered together with the results obtained from any LCA and E-LCC.

\section{Acknowledgements}

All H2020 REFRESH (Resource Efficient Food and dRink for Entire Supply cHain) partners who provided valuable feedback and comments. This paper has been selected as one of the best papers of the First joint SIDEA-SIEA Conference in Bisceglie/Trani (13-16 September 2017). It has been accepted for publication in this journal following the usual review process. 


\section{Authors' contributions}

All authors contributed equally to the conception and design of the study, in its framework and general assumptions. All authors contributed equally to the writing and review processes. FDM, JD, NU, and ML contributed to the modelling. FDM and MV collected the data related to school canteens and performed the analysis of the case study. KO and MV provided supervision and coordination. All authors read and approved the final manuscript.

\section{Funding}

This work was supported by the REFRESH (Resource Efficient Food and dRink for Entire Supply cHain) project, funded by the European Union Horizon 2020 Research and Innovation Programme under grant agreement no. 641933. More details on the REFRESH project can be found at http://eu-refresh.org.

\section{Availability of data and materials}

Not applicable

\section{Competing interests}

The authors declare that they have no competing interests.

\section{Author details}

${ }^{1}$ Department of Agricultural and Food Sciences, University of Bologna, Viale Giuseppe Fanin 50, 40127 Bologna, Italy.

${ }^{2}$ Research Institutes of Sweden, Agrifood and Bioscience, Forskningsbyn Ideon, Scheelevägen 27, SE-223 70 Lund, Sweden. ${ }^{3}$ University of Natural Resources and Life Sciences (BOKU), Muthgasse 107, A-1190 Wien, Vienna, Austria. ${ }^{4}$ Deloitte Sustainability, 185 Avenue Charle de Gaulle, 92200 Neuilly-sur-Seine, Paris, France.

Received: 13 August 2018 Accepted: 23 December 2019

Published online: 07 January 2020

\section{References}

Boschini M, Falasconi L, Giordano C, Alboni F (2018) Food waste in school canteens: a reference methodology for large-scale studies. J Clean Prod 182:1024-1032. https://doi.org/10.1016/j.jclepro.2018.02.040

Cerutti AK, Contu S, Ardente F, Donno D, Beccaro GL (2016) Carbon footprint in green public procurement: policy evaluation from acase study in the food sector. Food Policy 58:82-93. https://doi.org/10.1016/j.foodpol.2015.12.001

Corrado S, Ardente F, Sala S, Saouter E (2017) Modelling of food loss within life cycle assessment: from current practice towards a systematisation. J Clean Prod 140:847-859. https://doi.org/10.1016/j.jclepro.2016.06.050

Davis J, De Menna F, Unger N, et al (2017) Generic strategy LCA and LCC. Guidance for LCA and LCC focused on prevention, valorisation and treatment of side flows from the food supply chain

De Menna F, Dietershagen J, Loubiere M, Vittuari M (2018) Life cycle costing of food waste: a review of methodological approaches. Waste Manag 73:1-13. https://doi.org/10.1016/j.wasman.2017.12.032

De Menna F, Loubiere M, Dietershagen J, et al (2016) Methodology for evaluating LCC. Available at: https:/leu-refresh.org/ methodology-evaluating-life-cycle-costs-lcc-food-waste

EC (2013) Commission Recommendation of 9 April 2013 on the use of common methods to measure and communicate the life cycle environmental performance of products and organisations

EC JRC (2010) International Reference Life Cycle Data System (ILCD) Handbook - General guide for Life Cycle Assessment Detailed guidance. Publications Office of the European Union, Luxembourg

European Commission (2015) An EU action plan for the circular economy. European Commission, Bruxelles

European Parliament and Council (2008) Directive 2008/98/EC of the European Parliament and of the Council of 19 November 2008 on waste and repealing certain directives

FAO (2013) Food wastage footprint. Impacts on natural resources. Summary Report. FAO, Rome

FAO (2014) Food Wastage Footprint: Full cost-accounting. FAO, Rome

Garcia-Herrero L, De Menna F, Vittuari M (2019) Food waste at school. The environmental and cost impact of a canteen meal. Waste Manag 100:249-258. https://doi.org/10.1016/j.wasman.2019.09.027

Gruber LM, Brandstetter CP, Bos U et al (2016) LCA study of unconsumed food and the influence of consumer behavior. Int J Life Cycle Assess 21:773-784. https://doi.org/10.1007/s11367-015-0933-4

Gustavsson J, Cederberg C, Sonesson U et al (2011) Global food losses and food waste. Food and Agriculture Organization, Rome Hunkeler D, Lichtenvort K, Rebitzer G et al (2008) Environmental life cycle costing. CRC Press, Boca Raton

ISO (2006) ISO 14044:2006 Environmental management - life cycle assessment - requirements and guidelines. International Organization for Standardization, Geneva

Kummu M, de Moel H, Porkka M et al (2012) Lost food, wasted resources: global food supply chain losses and their impacts on freshwater, cropland, and fertiliser use. Sci Total Environ 438:477-489. https://doi.org/10.1016/j.scitotenv.2012.08.092

Martinez-Sanchez V, Tonini D, Møller F, Astrup TF (2016) Life-cycle costing of food waste management in Denmark: importance of indirect effects. Environ Sci Technol 50:4513-4523. https://doi.org/10.1021/acs.est.5b03536

Notarnicola B, Tassielli G, Renzulli PA et al (2016) Environmental impacts of food consumption in Europe. J Clean Prod. https://doi.org/10.1016/j.jclepro.2016.06.080

Swarr TE, Hunkeler D, Klöpffer W et al (2011) Environmental life-cycle costing: a code of practice. Int J Life Cycle Assess 16:389-391

Unger N, Davis J, Loubiere M, Östergren K (2016) Methodology for evaluating environmental sustainability. REFRESH Deliverable 5.1

Vittuari M, De Menna F, Pagani M (2016) The hidden burden of food waste: the double energy waste in Italy. Energies 9:660. https://doi.org/10.3390/en9080660

\section{Publisher's Note}

Springer Nature remains neutral with regard to jurisdictional claims in published maps and institutional affiliations. 